\title{
Molecular Observation of Constraint Release in Polymer Melts
}

\author{
M. Zamponi, A. Wischnewski, M. Monkenbusch, L. Willner, and D. Richter \\ Institut für Festkörperforschung, Forschungszentrum Jülich, 52425 Jülich, Germany \\ A. E. Likhtman \\ Department of Applied Mathematics, University of Leeds, Leeds LS2 9JT, United Kingdom \\ G. Kali and B. Farago \\ Institut Laue-Langevin, Avenue des Martyrs, 38042 Grenoble Cedex 9, France
}

(Received 9 March 2006; published 13 June 2006)

\begin{abstract}
The dynamics of binary polymer blends of few labeled long chains in successively shorter matrix chains has been investigated by neutron spin echo (NSE) spectroscopy. For the first time the effect of constraint release on the chain relaxation has been directly observed on a microscopic scale. Decreasing the matrix chain length reduces the topological confinement until unconfined Rouse motion is observed, when the matrix chains are too short to confine the long chain in a tube. Whereas an analytical description of the effect is not yet available, a new simulation based on the slip-link model shows perfect agreement with the NSE data over the full range of matrix molecular weights.
\end{abstract}

PACS numbers: 83.80.Sg, 61.12.Ex

The dynamics of linear polymer chains in the melt strongly depends on the chain length: For short, unentangled chains (and for any length at short times) the dynamics is determined by a balance of viscous and entropic forces which can be described by the Rouse model [1]. Here the chains interact solely by local friction with a heat bath, representing the neighboring chains. For long chains topological chain-chain interactions in terms of entanglements become important and are dominating the dynamical behavior. In the famous reptation model these constraints are described by a virtual tube which localizes a given chain and limits its motion to a one-dimensional Rouse motion inside the tube (local reptation) and a slow diffusive creep motion out of the tube (reptation) [2]. Neutron spin echo (NSE) spectroscopy is a powerful tool to explore the different dynamic regimes in polymer melts on a microscopic scale in space and time. While NSE experiments support the tube concept of topological confinement in long chain polymer systems [3,4], a close comparison of linear rheology data with predictions of the tube model indicates the existence of additional processes that release the topological confinement [5], such as fluctuating chain ends which escape the tube confinement (contour length fluctuations, CLF) and the relaxation of the tube itself (constraint release, CR). While CLF is an effect of the confined chain itself [6], CR stems from the movement of the chains building the tube, which of course undergo the same dynamical processes as the confined chain (see Fig. 1).

In this Letter we present a neutron spin echo study on binary polymer blends in order to investigate the influence of constraint release on the tube confinement by a systematic variation of the matrix molecular weight. This study demonstrates on a molecular level the increasing loss of confinement with the reduction of the host molecular weight and proves the role of CLF in the constraint release process. With a new slip-links model [7] we simulate the dynamics of polymer melts under consideration of CLF and CR and obtain a good description of the NSE data.

The different dynamic processes are reflected in the relaxation of the coherent single chain dynamic structure factor $S(q, t)$. This dynamic structure factor is directly measured by neutron spin echo spectroscopy on a sample containing a small fraction of protonated chains in a deuterated matrix. To separate the effect of CR from the CLF process, one has to consider a labeled chain which is long enough, such that end effects like CLF do not play a role. To control the CR effect by changing the matrix chain length, the concentration of the long chains was kept low enough in order to avoid any effects from the overlap of the long chains. To still get enough scattering intensity, 5\% of long labeled (protonated) polyethylene (PE) chains with a molecular weight of $M_{w}=36 \mathrm{~kg} / \mathrm{mol}$ have been mixed into successively shorter deuterated matrix chains with a

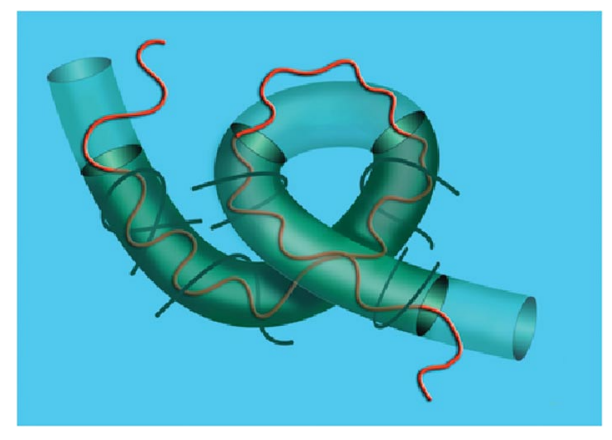

FIG. 1 (color). Schematic presentation of the CLF and CR mechanisms: chain end fluctuations lead to a shortening of the effective tube length, while the dissolving of entanglements allow chain motions beyond the initial tube constraints. 
molecular weight ranging from about 36 to $1 \mathrm{~kg} / \mathrm{mol}$ (see Table I) (entanglement molecular weight for $\mathrm{PE}$ is $M_{e}=$ $1-2 \mathrm{~kg} / \mathrm{mol})$.

The samples were obtained by anionic polymerization of butadiene with subsequent hydrogenation, respectively, deuteration. The dynamic structure factor of these binary blends has been investigated using the NSE spectrometer IN15, Institut Laue-Langevin, at a temperature of $T=509 \mathrm{~K}$, covering a momentum transfer range of $0.3 \mathrm{~nm}^{-1} \leq q \leq 1.15 \mathrm{~nm}^{-1}$. The moment transfer during scattering relates to the scattering angle $\theta$ and the neutron wavelength $\lambda$ as $q=\frac{4 \pi}{\lambda} \sin \left(\frac{\theta}{2}\right)$. Further measurements concentrating on the shorter time regime were performed at the NSE-FRJ2 spectrometer at the Research Centre Jülich.

Comparing the dynamic structure factor of the long chain in successive shorter matrix chains, a clear transition from confined reptationlike motion in long matrix chains to free Rouse motion in short matrix chains is observed. This can be well demonstrated in a Rouse scaling representation of the dynamic structure factor (Fig. 2). In the limit $q R_{e} \gg$ 1 ( $R_{e}$ the end-to-end distance of the chain) and for times shorter than the Rouse time $\tau_{R}$ (which both is fulfilled) the Rouse dynamic structure factor can be approximated by a function depending only on one parameter, the Rouse scaling variable $\sqrt{q^{4} W l^{4} t}$ [5], with the Rouse rate $W=$ $3 k_{B} T /\left(\xi l^{2}\right)$ ( $l$ Kuhn segment length, $\xi$ friction coefficient). In this representation all $S(q, t)$ need to collapse into one master curve. On the other hand, if topological constraints are evident a length scale comes into play (in the reptation model the tube diameter) and the such scaled dynamic structure factors split up for different $q$ values.

For the long chain in a matrix of the same molecular weight, a confinement as for infinite long chains is observed. This means that for a $36 \mathrm{~kg} / \mathrm{mol}$ chain neither CLF nor CR play a significant role in the accessible NSE time range, and the chain remains confined inside the tube. In a Rouse scaling plot as shown in Fig. 2 the dynamic structure factor splits for different $q$ values, displaying the topological constraints. The data are well described by de Gennes' reptation model with a tube diameter as for infinite long chains [lines, Eq. (2) in Ref. [4] ].

With decreasing matrix length $(12 \mathrm{~kg} / \mathrm{mol}, 6 \mathrm{~kg} / \mathrm{mol}$; Fig. 2) an increasing loss of confinement becomes visible

TABLE I. Molecular weight of the parent polybutadiene ( $h$ protonated, $d$ deuterated), polydispersity, number of simulated segments, and molecular weight dependent Rouse rate [8].

\begin{tabular}{lllrl}
\hline \hline Sample & $\frac{M_{w}}{\mathrm{~kg} / \mathrm{mol}}$ & $\frac{M_{w}}{M_{n}}$ & $N_{\text {sim }}$ & $\frac{W\left(M_{w}\right)}{W_{\infty}}$ \\
\hline $\mathrm{h} 36-$ & 35.9 & 1.025 & 166 & 1 \\
-d36 & 40 & 1.04 & 185 & 1 \\
-d12 & 12.4 & 1.025 & 57 & 1.05 \\
-d6 & 5.7 & 1.03 & 26 & 1.12 \\
-d2 & 2.3 & 1.06 & 11 & 1.35 \\
-d1 & 1 & 1.08 & 5 & 1.75 \\
\hline \hline
\end{tabular}

in the form of a stronger decay of the dynamic structure factor. This additional relaxation reflects the phenomenon of constraint release: the loosening of the tube confinement due to the motion of the surrounding chains. In the scaling representation the splitting for different $q$ values is still evident, but the reptation model fails to describe the data. For the $12 \mathrm{~kg} / \mathrm{mol}$ matrix a fit with the reptation model results in a wrong tube diameter, for the $6 \mathrm{~kg} / \mathrm{mol}$ matrix the model also fails qualitatively (dashed lines).

Eventually for a short chain matrix of only one entanglement length (about $2 \mathrm{~kg} / \mathrm{mol}$ ) the long labeled chain displays the characteristic Rouse scaling: obviously the matrix chains are too short to confine the long chain (Fig. 2). The data are qualitatively described by the Rouse model (lines), but some slight deviations are visible. For this binary blend the fitted Rouse parameter is $W l^{4}=$ $4.6 \mathrm{~nm}^{4} \mathrm{~ns}^{-1}$, which is even smaller than the value for long chain PE $W l^{4}=7 \mathrm{~nm}^{4} \mathrm{~ns}^{-1}$ [9]. To make sure that this is not an effect of the long chains forming entanglements, the concentration has been lowered to $2.5 \%$ with no effect on $S(q, t)$. Thus an overlap of the long chains can be excluded and the observed apparent reduction of the Rouse parameter must stem from remaining constraints due to the short chains.

Lowering the matrix chain length below the entanglement limit to $1 \mathrm{~kg} / \mathrm{mol}$, the $36 \mathrm{~kg} / \mathrm{mol}$ chain displays undisturbed Rouse motion with a fitted Rouse parameter of $W l^{4}=11.3 \mathrm{~nm}^{4} \mathrm{~ns}^{-1}$ close to the expected value $W l^{4}=12.3 \mathrm{~nm}^{4} \mathrm{~ns}^{-1}$ for the short chains when the molecular weight dependence is taken into account [8]. Although observing the dynamics of the long chain the segmental friction of the short chains is measured.

The key question now is which dynamical processes underlie the CR process and lead to the gradual loss of confinement with decreasing matrix chain length. The

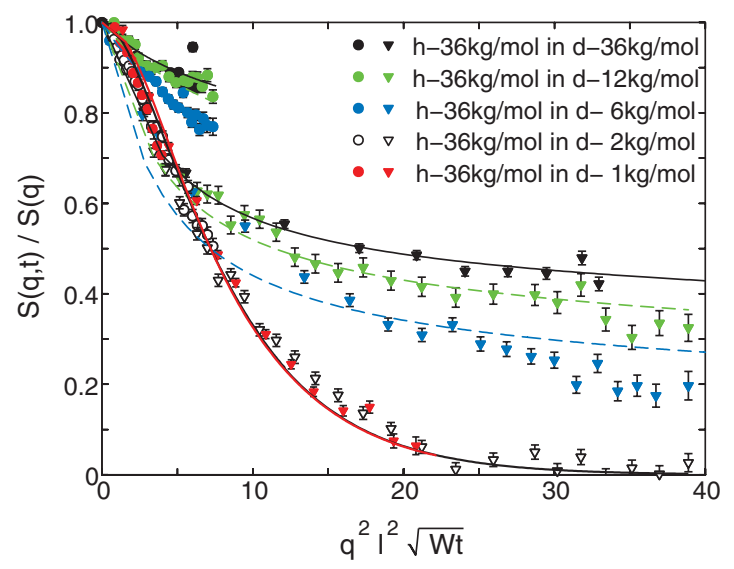

FIG. 2 (color). Dynamic structure factor of a long labeled chain $\left(M_{w}=36 \mathrm{~kg} / \mathrm{mol}\right)$ in different shorter matrix chains $\left(M_{w}=36,12,6,2,1 \mathrm{~kg} / \mathrm{mol}\right.$ as indicated in the plot) in a Rouse scaling representation for two different $q$ values (circles $0.5 \mathrm{~nm}^{-1}$, triangles $1.15 \mathrm{~nm}^{-1}$ ). For an explanation of the lines, see text. 
reptation time of the matrix chains $\left[\tau_{d}(12 \mathrm{~kg} / \mathrm{mol}) \approx\right.$ $\left.5000 \mathrm{~ns}, \tau_{d}(6 \mathrm{~kg} / \mathrm{mol}) \approx 500 \mathrm{~ns}\right]$ is far beyond the experimental time range. This holds even more for the characteristic times for constraint release $\tau_{\mathrm{CR}}=$ $\tau_{e}\left(M_{\text {long }} / M_{e}\right)^{2}\left(M_{\text {matrix }} / M_{e}\right)^{3}$ [10] which are based on a Rouse dynamic of the tube confining the long chain. They are in the range of hundreds of microseconds. But even for the $12 \mathrm{~kg} / \mathrm{mol}$ matrix, which is well entangled, the effect of CR is considerable. This fact already demonstrates that for an estimation of the characteristic time scale all dynamical processes, which are known to determine the segmental motion, have to be taken into account. These processes include next to the reptational creep also CLF of the chain ends [11]. In contrast to the center of mass, the segmental mean square displacement is significant in the experimental time range. Using the experimental parameters it can be estimated within the Rouse model to $\left\langle r_{\text {segm }}^{2}\right\rangle=\sqrt{\frac{4}{\pi} W l^{4} t}$, i.e., after the initial decay of $S(q, t)$ $(20-30 \mathrm{~ns}) \sqrt{\left\langle r_{\text {segm }}^{2}\right\rangle}=3.7-4.0 \mathrm{~nm}$.

We can now design an experiment to separate the different dynamic processes: For a long chain in a short matrix CLF is negligible; therefore the effect of CR of the matrix chains can be observed. On the other hand, for the dynamics of a short chain in a long matrix, the CLF of the short chain are dominating: no $\mathrm{CR}$ of the matrix chains can occur. Comparing the dynamic structure factor of such a corresponding pair of samples the contribution from CLF and CR of the shorter chain can be separated. Figure 3 shows the result for a $12 \mathrm{~kg} / \mathrm{mol}$ chain in a $36 \mathrm{~kg} / \mathrm{mol}$ matrix and vice versa. The long chain relaxation by constraint release of the short matrix is obviously identical to the relaxation of the confined short chain by contour length fluctuations. That is, in the $12 \mathrm{~kg} / \mathrm{mol}$ matrix the CR visible in the long chain dynamics may be traced solely to the CLF of the matrix chains. Thus, this experiment shows that even CLF alone can cause the CR effect. The CLF effect on the self-diffusion is an important subject under controversy [12], but the self-diffusion is negligible

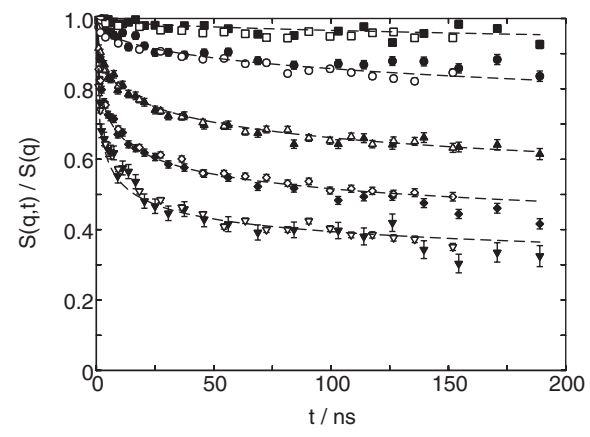

FIG. 3. Dynamic structure factor of a $36 \mathrm{~kg} / \mathrm{mol}$ chain in a $12 \mathrm{~kg} / \mathrm{mol}$ matrix (solid symbols) and vice versa (open symbols). $Q$ values (in $\mathrm{nm}^{-1}$ ): squares 0.3 , circles 0.5 , up-pointing triangles 0.77 , diamonds 0.96 , and down-pointing triangles 1.15 . Lines are just guides for the eye. in the time scale examined here and thus this effect is out of the scope of this Letter.

An analytical solution for the CR effect is difficult since it is a many chain problem. Therefore, in order to describe the series of experimental data quantitatively, we use a recently developed simple self-consistent slip-links model which can be solved by computer simulation. The salient features of the model will be recapitulated here; more details can be found in [7]. A Rouse chain in three dimensions is modeled by $N+1$ beads which are connected by springs, forming a chain of $N$ segments of length $l$. The only interaction with other chains is described by the segmental friction $\xi$ of the chain with a heat bath. The topological constraints are then introduced by a parabolic mean field potential which confines the monomers. This potential can be visualized by $Z=N / N_{e}$ slip links which are fixed by springs of the strength $3 k_{B} T /\left(N_{s} l^{2}\right)$ to anchor points. $N_{e}$ is the average number of segments between slip links and $N_{s}$ the number of segments of the anchor springs. The slip links can move along the chain with a friction $\xi_{s}$ to allow the chain to reptate. From the simulated monomer trajectories the single chain dynamic structure factor can then be easily obtained as the pair correlation function of the monomers.

Within this model the reptation limiting processes CLF and $\mathrm{CR}$ are easily implemented without introducing any additional parameter. If a slip link reaches the end of a chain, it disappears, which simulates the effect of CLF. For describing CR an ensemble of independent chains is simulated, which interact only by the following mechanism: pairwise, 2 slip links on different chains are randomly chosen and associated, so that if one slip link disappears the corresponding second slip link is also removed. A new pair of slip links is then reintroduced on two arbitrary chains, one at the end of a chain, the other one at a random place of the other chain.

To map the simulation results onto the experimental data the length and time scale of the simulation model have to be rescaled to the "real" parameters. The elementary time scale for an unconfined polymer chain in the melt is the Rouse time $\tau_{R}=N^{2} \tau_{0}=N^{2} /\left(\pi^{2} W\right)$. The length scale of the chain is the end-to-end distance $R_{e}^{2}=N l^{2}$. The respective scales in the simulation are then $l_{\text {sim }}=\sqrt{N_{0} l^{2}}$ and $\tau_{\text {sim }}=N_{0}^{2} /\left(\pi^{2} W\right)$, where $N_{0}=M_{0}^{\text {sim }} / M_{0}^{\text {real }}$ gives the chosen coarse graining of the model.

The only unknown parameters are the number and strength of the slip links $\left(N_{e}\right.$ and $\left.N_{s}\right)$ and their friction $\xi_{s}$ along the chain. This artificial friction had to be introduced to avoid zero escape time of the slip links from the chain and is chosen to be much smaller than the friction of the monomers $\left(\xi_{s}=0.01 \xi\right)$, so that the results only marginally depend on this value. The other two parameters replace the tube diameter in the reptation model. As was shown in Ref. [7], the results depend only on the combination of $N_{e}$ and $N_{s}$, thus making this combination a single parameter describing entanglements. 

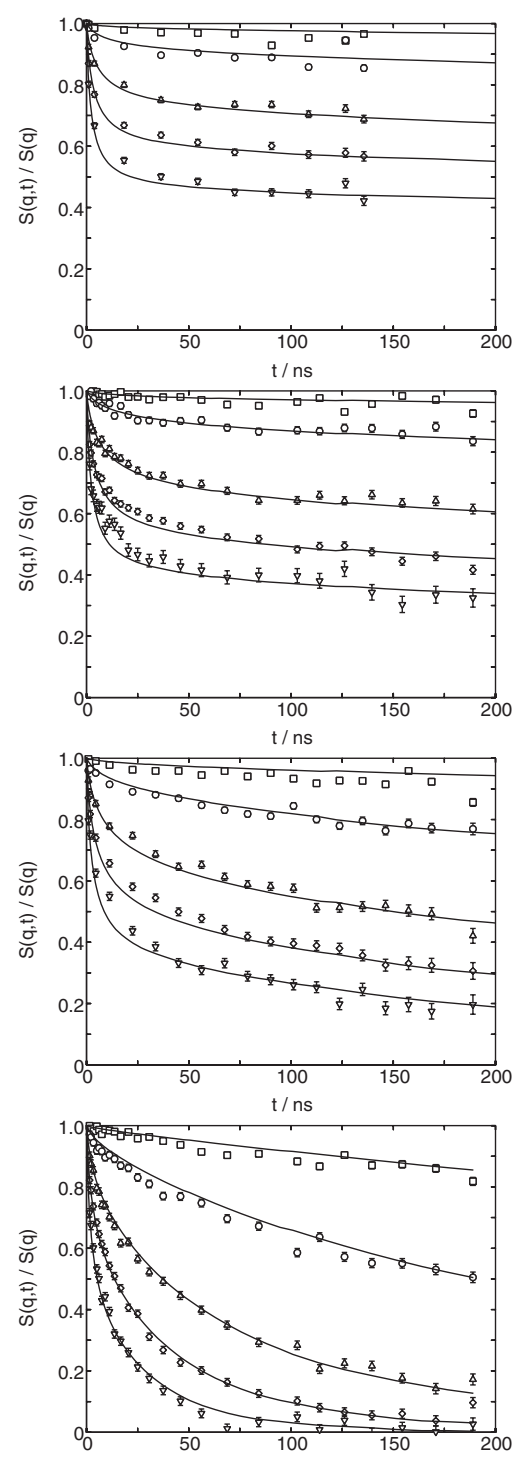

$\mathrm{t} / \mathrm{ns}$

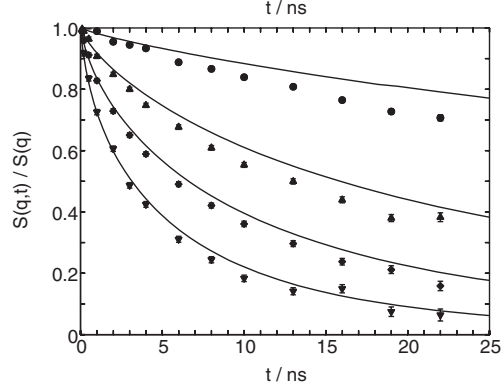

FIG. 4. Dynamic structure factor from a $M_{w}=36 \mathrm{~kg} / \mathrm{mol} \mathrm{PE}$ chain in successive shorter matrix chains $\left(M_{w}=\right.$ $36,12,6,2,1 \mathrm{~kg} / \mathrm{mol}$ from top to bottom) for the different $q$ values (values as in Fig. 3). Lines show the simulation results.

The coarse graining was chosen such that further refinement did not change $S(q, t)$ within the observation window. Then $N_{e}$ and $N_{s}$ are selected such that the simulated structure factor describes well the NSE data of a nearly infinite long $\left(M_{w}=190 \mathrm{~kg} / \mathrm{mol}\right)$ homopolymer PE melt. With the same set of parameters also other homopolymer
PE melts over a large range of molecular weights can be described [7]. To compare now data and simulation results for the binary blends the same parameters as for the homopolymer melts are used. The ensemble of simulated chains consist of chains with two different chain lengths corresponding to the actual samples, where the dynamic structure factor of the long chain (which is labeled in the experiments) is calculated.

As can be seen in Fig. 4 very good agreement of simulation and NSE data for all the different blends over the whole $q$ range is achieved. Without introducing any new parameters or adjusting the parameters used for the homopolymer melts the effect of constraint release is quantitatively captured in the simulation model. Because of the molecular weight dependence of the Rouse rate, the simulation time scale has to be rescaled. For the comparison as shown in Fig. 4 the time scale was recalculated according to the molecular weight dependent changes of the Rouse rate (see Table I). The simulation results clearly reproduce the transition from confined reptational motion to free Rouse motion.

To conclude, the influence of the surrounding matrix chains on the topological confinement of long polymer chains has been systematically investigated by neutron spin echo spectroscopy. The effect of constraint release has been observed for the first time directly on a molecular level in space and time. It has been experimentally proven that already CLF of the matrix chains alone can lead to CR. The simulation of the slip-link model gives a consistent description of the experimental data over the whole range of matrix molecular weights.

[1] P. E. Rouse, J. Chem. Phys. 21, 1272 (1953).

[2] P. G. de Gennes, J. Phys. (Paris) 42, 735 (1981).

[3] P. Schleger, B. Farago, C. Lartigue, A. Kollmar, and D. Richter, Phys. Rev. Lett. 81, 124 (1998).

[4] A. Wischnewski, M. Monkenbusch, L. Willner, D. Richter, A.E. Likhtman, T.C.B. McLeish, and B. Farago, Phys. Rev. Lett. 88, 058301 (2002).

[5] M. Doi and S. Edwards, The Theory of Polymer Dynamics (Oxford University Press, Oxford, 1986).

[6] M. Zamponi, M. Monkenbusch, L. Willner, A. Wischnewski, B. Farago, and D. Richter, Europhys. Lett. 72, 1039 (2005).

[7] A. E. Likhtman, Macromolecules 38, 6128 (2005).

[8] D.S. Pearson, G. Ver Strate, E. von Meerwall, and F. C. Schilling, Macromolecules 20, 1133 (1987).

[9] D. Richter, B. Farago, R. Butera, L. J. Fetters, J. S. Huang, and B. Ewen, Macromolecules 26, 795 (1993).

[10] T. C. B. McLeish, Adv. Phys. 51, 1379 (2002).

[11] M. Rubinstein and R. H. Colby, J. Chem. Phys. 89, 5291 (1988).

[12] S. Wang, E. D. von Meerwall, S.-Q. Wang, A. Halasa, W.-L. Hsu, J.P. Zhou, and R. P. Quirk, Macromolecules 37, 1641 (2004). 